PAPER • OPEN ACCESS

\title{
Quantum principle of relativity
}

To cite this article: Andrzej Dragan and Artur Ekert 2020 New J. Phys. 22033038

View the article online for updates and enhancements. 


\section{New Journal of Physics}

The open access journal at the forefront of physics
Published in partnership with: Deutsche Physikalische

Gesellschaft and the Institute of Physics

\section{PAPER}

\section{Quantum principle of relativity}

\section{OPEN ACCESS}

\section{RECEIVED}

2 December 2019

REVISED

24 January 2020

ACCEPTED FOR PUBLICATION

17 February 2020

PUBLISHED

24 March 2020

Original content from this work may be used under the terms of the Creative

Commons Attribution 4.0 licence.

Any further distribution of this work must maintain attribution to the author(s) and the title of the work, journal citation and DOI.

\author{
Andrzej Dragan ${ }^{1,2}$ and Artur Ekert ${ }^{2,3}$ \\ 1 Institute of Theoretical Physics, University of Warsaw, Pasteura 5, 02-093 Warsaw, Poland \\ Centre for Quantum Technologies, National University of Singapore, 3 Science Drive 2, 117543 Singapore, Singapore \\ Mathematical Institute, University of Oxford, Woodstock Road, Oxford OX2 6GG, United Kingdom \\ E-mail: dragan@fuw.edu.pl
}

Keywords: quantum theory, special relativity, quantum indeterminacy

\section{Abstract}

Quantum mechanics is an incredibly successful theory and yet the statistical nature of its predictions is hard to accept and has been the subject of numerous debates. The notion of inherent randomness, something that happens without any cause, goes against our rational understanding of reality. To add to the puzzle, randomness that appears in non-relativistic quantum theory tacitly respects relativity, for example, it makes instantaneous signaling impossible. Here, we argue that this is because the special theory of relativity can itself account for such a random behavior. We show that the full mathematical structure of the Lorentz transformation, the one which includes the superluminal part, implies the emergence of non-deterministic dynamics, together with complex probability amplitudes and multiple trajectories. This indicates that the connections between the two seemingly different theories are deeper and more subtle than previously thought.

\section{All inertial observers}

The full mathematical structure of the Lorentz transformations contains both subluminal and superluminal terms. The superluminal part is usually discarded, on the premise that it makes no physical sense, and, as a consequence, a familiar classical picture of a particle moving along a well defined path is obtained. Here we show that if we retain the superluminal terms, and take the resulting mathematics of the Lorentz transformation seriously, then the notion of a particle moving along a single path must be abandoned and replaced by a propagation along many paths, exactly like in quantum theory.

The generalised Lorentz transformation can be derived in few simple steps [1]. Consider a classical $1+1$ dimensional case (the $1+3$ case will be discussed later) with an inertial frame $\left(t^{\prime}, x^{\prime}\right)$ moving with the velocity $V$ relative to the frame $(t, x)$. We seek the most general form of the coordinate transformation between these frames that is consistent with the Galilean principle of relativity. It has to be a linear transformation, so that no point in spacetime is singled out, and its coefficient must depend only on the relative velocity $V$. The inverse transformation involves a sign flip in the velocity $V$ :

$$
\begin{aligned}
x^{\prime} & =A(V) x+B(V) t, \\
x & =A(-V) x^{\prime}+B(-V) t^{\prime},
\end{aligned}
$$

where $A(V)$ and $B(V)$ are unknown functions we wish to determine. The origin of the primed frame $x^{\prime}=0$, is moving according to the equation $x=V t$. Putting that into (1) we obtain $\frac{B(V)}{A(V)}=-V$, which allows us to write (1) as:

$$
\begin{aligned}
x^{\prime} & =A(V)(x-V t), \\
t^{\prime} & =A(V)\left(t-\frac{A(V) A(-V)-1}{V^{2} A(V) A(-V)} V x\right) .
\end{aligned}
$$

At this stage all we can say about $A(V)$ is that it is either a symmetric or antisymmetric function of its argument. This is because a discrete change of sign of any spacetime coordinate in the unprimed frame should result in a 
discrete sign change in the transformation formulas (2). But since such a sign flip also affects the sign of velocity $V$, the quantity $A(V)$ has to be either symmetric or antisymmetric.

In order to determine $A(V)$ uniquely, consider three inertial frames $(t, x),\left(t^{\prime}, x^{\prime}\right)$, and $\left(t^{\prime \prime}, x^{\prime \prime}\right)$ and let the primed frame move with the velocity $V_{1}$ relative to the unprimed frame, and let the double-primed frame move with the velocity $V_{2}$ relative to the primed one. By iterating (2) we obtain the velocity of the double-primed observer relative to the unprimed one:

$$
x^{\prime \prime}=A\left(V_{1}\right) A\left(V_{2}\right) x\left(1+V_{1} V_{2} \frac{A\left(V_{1}\right) A\left(-V_{1}\right)-1}{V_{1}^{2} A\left(V_{1}\right) A\left(-V_{1}\right)}\right)-A\left(V_{1}\right) A\left(V_{2}\right)\left(V_{1}+V_{2}\right) t .
$$

Looking at the first equation in (2) we see that we can compute $V$ by calculating the ratio between the coefficient at $t$ and the coefficient at $x$ (and reversing the sign). Applying this rule to (3) we obtain:

$$
V=\frac{V_{1}+V_{2}}{1+V_{1} V_{2} \frac{A\left(V_{1}\right) A\left(-V_{1}\right)-1}{V_{1}^{2} A\left(V_{1}\right) A\left(-V_{1}\right)}} .
$$

Notice that interchanging $V_{1} \leftrightarrow-V_{2}$ in (4) should result in a velocity of the unprimed observer relative to the double-primed observer, which is $-V$ :

$$
-V=\frac{-V_{2}-V_{1}}{1+V_{2} V_{1} \frac{A\left(-V_{2}\right) A\left(V_{2}\right)-1}{V_{2}^{2} A\left(-V_{2}\right) A\left(V_{2}\right)}} .
$$

Equating (5) with (4) brings us to

$$
\frac{A\left(V_{1}\right) A\left(-V_{1}\right)-1}{V_{1}^{2} A\left(V_{1}\right) A\left(-V_{1}\right)}=\frac{A\left(V_{2}\right) A\left(-V_{2}\right)-1}{V_{2}^{2} A\left(V_{2}\right) A\left(-V_{2}\right)},
$$

for any $V_{1}$ and $V_{2}$. This can be satisfied only if both sides of the equation are equal to some constant $K$ :

$$
\frac{A(V) A(-V)-1}{V^{2} A(V) A(-V)}=K,
$$

which sets a constraint on possible functions $A(V)$ appearing in (2).

For the symmetric case, $A(-\mathrm{V})=A(V)$, the condition (7) gives $A(V)= \pm \frac{1}{\sqrt{1-K V^{2}}}$. Choosing the sign such that for $V \rightarrow 0$ we get $x^{\prime} \rightarrow x$, we retrieve:

$$
\begin{aligned}
x^{\prime} & =\frac{x-V t}{\sqrt{1-K V^{2}}}, \\
t^{\prime} & =\frac{t-K V x}{\sqrt{1-K V^{2}}} .
\end{aligned}
$$

The new constant $K$ remains unknown. The case of $K=0$ corresponds to the Galilean universe, the case of $K>0$ leads to relativistic spacetime as we know it. The last case of $K<0$ corresponds to an Euclidean spacetime with one of the dimensions stretched by an extra factor of $\sqrt{|K|}$ and the derived transformation being just a regular rotation. From now on, we pick $K=\frac{1}{c^{2}}$, which brings us to the Lorentz transformation, well-behaved for velocities $V<c$.

For the anti-symmetric case $A(-\mathrm{V})=-\mathrm{A}(V)$ the constraint (7) gives $A(V)= \pm \frac{V /|V|}{\sqrt{V^{2} / c^{2}-1}}$, which is wellbehaved for $V>c$ and leads to the following transformation [2, 5]:

$$
\begin{aligned}
x^{\prime} & = \pm \frac{V}{|V|} \frac{x-V t}{\sqrt{V^{2} / c^{2}-1}}, \\
t^{\prime} & = \pm \frac{V}{|V|} \frac{t-V x / c^{2}}{\sqrt{V^{2} / c^{2}-1}} .
\end{aligned}
$$

So far, we have only used the Galilean principle of relativity, which puts no restrictions on possible velocities of the observer. Both the solutions (8) and (9) preserve the constancy of the speed of light. In order to get rid of the second branch of solutions (9), we have to introduce additional physical assumptions that rule them out. We choose not to do so. Instead, we will investigate the physical consequences of these extra solutions.

Two comments are in order. First, the sign in front of the equations (9) cannot be uniquely determined, because no $V \rightarrow 0$ limit exists. The choice of the sign must remain a matter of convention, and from now on we will pick the negative sign. Second, both branches of solutions form a symmetry group only in the considered $1+1$ dimensional scenario. This is not the case in the $1+3$ dimensional case [3], therefore we will carefully discuss this case separately in section 5 . For now we stick to the $1+1$ scenario, in which equations (9) describe a hyperbolic rotation by the angle $\in\left(\frac{\pi}{4}, \frac{3 \pi}{4}\right)$. 

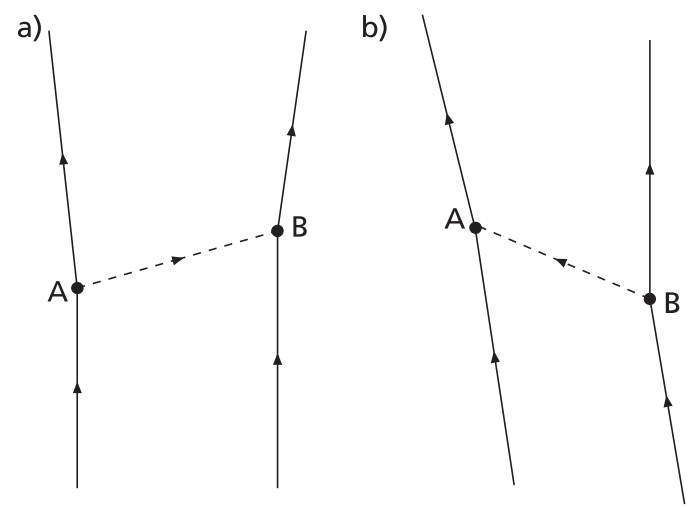

Figure 1. Spacetime diagrams of a process of sending a superluminal particle as seen by two inertial observers (time is vertical, space is horizontal): (a) particle emitted from A and absorbed in B, (b) the same process observed in a different inertial frame.

\section{Indeterministic behavior}

So far we have shown that the Galilean principle of relativity alone leads to two branches of coordinate transformations corresponding to subluminal and superluminal families of observers. In the $1+1$ dimensional scenario these branches are indistinguishable, which means that a particle at rest with respect to an observer belonging to one of the branches will be considered superluminal by the observer belonging to the other branch -being superluminal is relative. Let us now show that relativistic, local, and deterministic description of fundamental processes is no longer possible.

Suppose that a superluminal particle observed by some inertial observer was emitted from a source particle at the event $A$ and then absorbed at some later time by the identical target particle at the event $B$ - see figure 1(a). The same process observed from a reference frame moving with a relative subluminal velocity is depicted in figure 1(b), where $B$ becomes the emission of the superluminal particle, and $A$ the absorption.

Let us focus on the first frame shown in figure 1(a) and assume that the moment of emission at A could be predicted using a local and deterministic mode of description. In other words, let us assume that the past worldline of the source particle prior to the event A contains locally all the information necessary to predict the exact moment of emission of a superluminal particle at A. Or using the Einsteinian language, there is an element reality to it. On the other hand someone holding the target particle $B$ cannot predict the moment of the absorption at $B$ based only on local measurements of the particle $B$ prior to the event. Now, let us change the reference frame and study the same scenario from the perspective of the observer moving with some subluminal speed, as depicted in figure 1(b). Let us ask the question: what caused the emission of the superluminal particle at the event $B$ ?

We could say that the cause of the event B takes place in the distant world line of the particle A. Possibly at a later time than the event $B$ itself. However, if we seek a deterministic and local mode of description, i.e. trying to determine the moment of emission at $B$ only by a local measurement on the particle $B$, it is clearly impossible. We have already assumed that the past world-line of the particle $B$ carries no information about the time of the event $B$. In practice, the observer having only access to the local properties of the particle $B$ can only conclude that the emission at $B$ was be completely spontaneous and fundamentally unpredictable.

We have previously assumed that the cause of the emission of the superluminal particle at $A$ (in the first reference frame) was determined by the past world-line of $A$. This assumption leads, however to a preferred reference frame, in which a local deterministic mode of description is possible, while it remains impossible in other frames. It becomes especially clear if both particles $A$ and $B$ are identical. To preserve the Galilean principle of relativity, we have to abandon our assumption that the emission at $A$ in the first frame could be determined by a local process. As a result we conclude that no relativistic, local and deterministic description of the emission of a superluminal particle is possible in any inertial frame. If such an emission was to take place, it would have to appear completely random to any inertial observer. If we had a source of superluminal particles at our disposal, we would not be able to use it to send any information because we would not be able to control the emission rate using any local operations.

Non-deterministic behavior is not only a property of superluminal particles for the same applies to subluminal particles. Consider a decay of such a particle into a pair of other subluminal particles, as depicted in figure 2(a). Let us picture the same process as seen by the infinitely fast moving inertial observer, for which the transformation (9) reduces to: 
a)

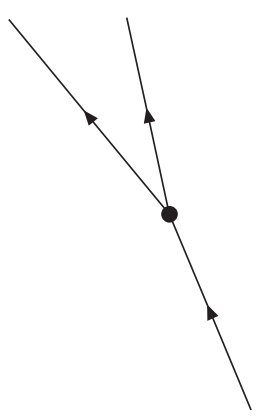

b)

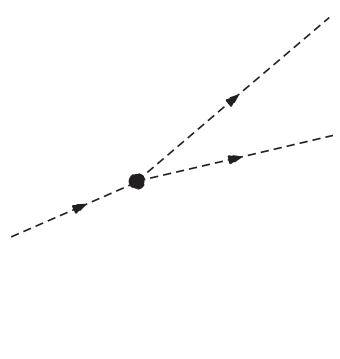

Figure 2. A spacetime diagram of a decay of a subluminal particle into a pair of subluminal particles (time is vertical, space is horizontal): (a) in a subluminal reference frame, (b) in a superluminal reference frame.

a)

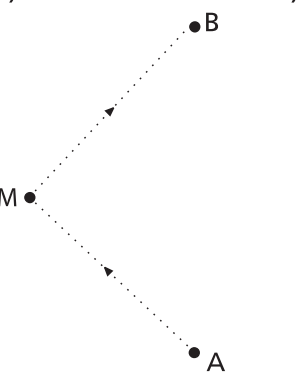

b)

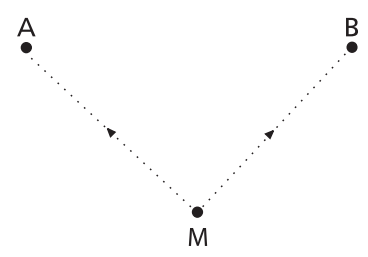

Figure 3. A spacetime diagram of a luminal particle (dotted line) reflected from a mirror (time is vertical, space is horizontal): (a) in a subluminal reference frame, (b) in a superluminal reference frame.

$$
\begin{aligned}
x^{\prime} & =c t, \\
c t^{\prime} & =x .
\end{aligned}
$$

For such a frame, the considered decay process is depicted in figure 2(b), where all particles are superluminal and henceforth, the decay cannot be described using any local and deterministic theory, as we have shown earlier. By invoking the Galilean principle of relativity we conclude that the same must be the case for any subluminal reference frame.

\section{Multiple paths}

Another property of the quantum theory, besides it being non-deterministic, is the fact that a particle that is not observed behaves as if it was moving along multiple trajectories at once, which is best shown in interference experiments. But once the particle is observed it can only be detected at one of the locations. Now, let us show how this follows from the Galilean principle of relativity involving both families of inertial observers (8) and (9).

Consider a photon emitted from a source at $A$, reflected from a mirror $M$ and then received at $B$, as shown in figure 3(a). Suppose that we want to detect the photon by placing detectors at its path. If a detector placed at the path A-M absorbs the photon, then a similar detector placed at the path $\mathrm{M}-\mathrm{B}$ will not register anything, because the photon has been absorbed earlier. Similarly, if a detector at M-B absorbed the photon, then certainly, the photon could not have been detected at the path A-M. Now let us analyze the same scenario from an infinitely fast moving reference frame by applying equations (10). In this reference frame the photon is traveling from $M$ towards $A$ and $B$ along two paths, but if we try to detect it using a pair of detectors placed at $M-A$ and $M-B$ then only one of them can absorb the photon. However as long as we do not make any observation, the motion of the photon is characterized by two simultaneous paths, not one.

As we can see, even if we start with an idea of a classical particle moving along a single path, it is only a matter of a change of the reference frame to arrive at a scenario involving more than one path.

Consider a process depicted in figure 4(a) in which a particle emitted in $\mathrm{A}$ is scattered in $\alpha$, where it starts to follow two paths at once towards B and B. The same process viewed from the infinitely fast moving frame will involve the particle following three paths at once. This concept can be iterated leading to scenarios involving 

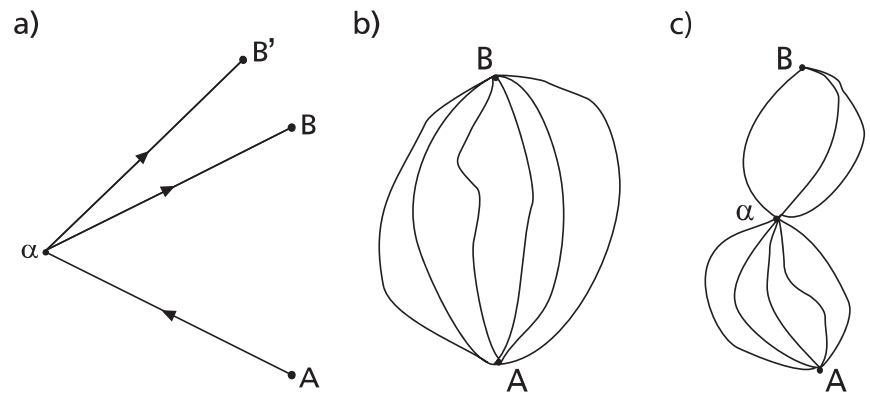

Figure 4. (a) Particle emitted in $A$ is scattered at $\alpha$ into a motion along two paths towards $B$ and $B^{\prime}$ at once. (b) and (c) multiple paths connecting two spacetime points, $\mathrm{A}$ and $\mathrm{B}$, in two possible settings.

multiple paths at once. Once both branches of transformations (8) and (9) are involved, a classical description of a particle always moving along a single trajectory becomes inconsistent with the Galilean principle of relativity.

\section{Probability amplitudes}

Let us go back to the orthodox setting of subluminal particles being observed by subluminal observers. Relativistic theories operate on notions that do not change under Lorentz transformations. We will therefore investigate, what are relativistically invariant quantities that describe a particle motion between two points, $A$ and $B$ that have properties of probability. The simplest relativistic invariant characterizing a single path is its relativistic length, i.e. the proper time:

$$
\phi \sim \int_{\mathrm{A}}^{\mathrm{B}} \sqrt{1-v^{2} / c^{2}} \mathrm{~d} t \sim \int_{\mathrm{A}}^{\mathrm{B}}(E \mathrm{~d} t-p \mathrm{~d} x)
$$

where $E \sim \frac{1}{\sqrt{1-v^{2} / c^{2}}}$ is the energy of the particle and $p \sim \frac{v}{\sqrt{1-v^{2} / c^{2}}}$ is its momentum. We will choose the proportionality constant such that the relativistic invariant $\phi$ is dimensionless and will be referred to as the phase along the path. When multiple paths are involved, as shown in figure 4(b), a relativistic invariant characterizing such a diagram must be a function of phases along all individual paths, $\mathcal{P}^{(n)}\left(\phi_{1}, \phi_{2}, \ldots, \phi_{n}\right)$, where $\mathcal{P}$ is a smooth function and $n$ is the number of possible paths. We will be interested in the question, what are reasonable functions $\mathcal{P}$ to consider. It turns out that the family of such functions is not too vast.

First of all, our choice of the way we label individual paths has no physical significance, therefore a reasonable function $\mathcal{P}$ should be a symmetric function of its arguments:

$$
\mathcal{P}^{(n)}\left(\phi_{1}, \phi_{2}, \ldots, \phi_{n}\right)=\mathcal{P}^{(n)}\left(\phi_{\pi(1)}, \phi_{\pi(2)}, \ldots, \phi_{\pi(n)}\right),
$$

where $\pi$ is an any permutation of an $n$-element set. Second of all, we choose our description to be completely time-symmetric, which boils down to:

$$
\mathcal{P}^{(n)}\left(\phi_{1}, \phi_{2}, \ldots, \phi_{n}\right)=\mathcal{P}^{(n)}\left(-\phi_{1},-\phi_{2}, \ldots,-\phi_{n}\right) .
$$

Consider a special type of motion, in which all trajectories intersect at a single point $\alpha$, as shown in figure 4(c). Suppose that the events $\mathrm{A}$ and $\alpha$ are linked by $n$ different paths characterized by phases $\phi_{1}, \ldots, \phi_{n}$, while $\alpha$ and B are interlinked by $m$ paths characterized by phases $\xi_{1}, \ldots, \xi_{m}$. The total number of paths connecting $A$ and $B$ is equal to $n m$ and since the phases are additive, such motion involves sums of phases $\phi_{i}+\xi_{j}$. Therefore the invariant function for such a motion is $\mathcal{P}^{(n m)}\left(\phi_{1}+\xi_{1}, \phi_{1}+\xi_{2}, \phi_{1}+\xi_{3}, \ldots, \phi_{n}+\xi_{m}\right)$.

Our third and final condition captures the fact that the invariant quantity we are trying to establish should have properties of probability. Since the probability for the particle to travel from $A$ to $B$ should be a product of probabilities for the particle to travel from $\mathrm{A}$ to $\alpha$ and then from $\alpha$ to $\mathrm{B}$, we impose the third condition:

$$
\mathcal{P}^{(n m)}\left(\phi_{1}+\xi_{1}, \phi_{1}+\xi_{2}, \phi_{1}+\xi_{3}, \ldots, \phi_{n}+\xi_{m}\right)=\mathcal{P}^{(n)}\left(\phi_{1}, \phi_{2}, \ldots, \phi_{n}\right) \mathcal{P}^{(m)}\left(\xi_{1}, \xi_{2}, \ldots, \xi_{m}\right) .
$$

A special case that satisfies all the conditions (12)-(14) has the form:

$$
\mathcal{P}^{(n)}\left(\phi_{1}, \phi_{2}, \ldots, \phi_{n}\right)=\frac{1}{n^{\beta}}\left(\mathrm{e}^{\alpha \phi_{1}}+\mathrm{e}^{\alpha \phi_{2}}+\ldots+\mathrm{e}^{\alpha \phi_{n}}\right)^{\gamma}\left(\mathrm{e}^{-\alpha \phi_{1}}+\mathrm{e}^{-\alpha \phi_{2}}+\ldots+\mathrm{e}^{-\alpha \phi_{n}}\right)^{\gamma},
$$

where $\alpha, \beta$ and $\gamma$ are arbitrary constants. We show elsewhere [5] that a general form of the function $\mathcal{P}$ is a multi product of special solutions (15) with any constants $\alpha, \beta$ and $\gamma$.

For the infinite number of trajectories all these invariants tend to diverge or go to zero if $\gamma \neq 0$. The invariant can remain finite for the infinite number of paths only if the constant $\alpha$ takes a purely imaginary value. As a consequence, a relativistically invariant description of the scenario, in which a particle is moving along 
(infinitely) many possible paths involves the quantity: $\langle\mathrm{B} \mid \mathrm{A}\rangle \sim \sum_{k} \mathrm{e}^{\mathrm{i}|\alpha| \phi_{k}}$, known as the (complex) probability amplitude, for which the proportionality constant can be established based on the normalization condition.

This result shows that relativistic invariance and symmetry requirements lead to the characterization of the probability-like quantities that are based on a sum of complex exponential functions that we call probability amplitudes.

Let us mention in passing that the proportionality constant missing from equation (11) is the inverse of the Planck's constant, $\hbar^{-1}$ and its value cannot be determined $a$ priori by any known methods, just like the constant $K=\frac{1}{c^{2}}$ appearing in equation (7). Let us also add that here we have only considered a freely moving point-like particle propagating along multiple paths and found that its dynamics can be characterised by probability amplitudes in the form of plain waves. If we were to look for a dynamical equation governing the evolution of these amplitudes, we would have a choice of either a Schrödinger, a Klein-Gordon, or a Dirac equation, because all of them have plain waves as the elementary solutions.

\section{$5.1+3$ dimensional case}

Situation becomes more interesting for the $1+3$ dimensional case. It was shown that the smallest group involving both subluminal and superluminal four-dimensional transformations is $S L(4, \mathbb{R})$ [3]. This cannot be a symmetry group, because it involves transformations such as direction-dependent time dilation, which are not observed [3]. Therefore superluminal transformations in $1+3$ dimensional spacetime should not be symmetries. According to one interpretation [4], in contrast to the $1+1$ dimensional case, the family of superluminal observers can be distinguished from the subluminal observers and therefore being superluminal is not a relative notion anymore. The spacetime interval in the moving frame $\left(t^{\prime}, x^{\prime}, y^{\prime}, z^{\prime}\right)$ :

$$
c^{2} \mathrm{~d} t^{2}-\mathrm{d} x^{2}-\mathrm{d} y^{2}-\mathrm{d} z^{2}=-c^{2} \mathrm{~d} t^{\prime 2}+\mathrm{d} x^{\prime 2}-\mathrm{d} y^{\prime 2}-\mathrm{d} z^{\prime 2}
$$

has a non-Euclidean spatial component $\mathrm{d} x^{\prime 2}-\mathrm{d} y^{\prime 2}-\mathrm{d} z^{\prime 2}$, which can be physically discriminated from the Euclidean space of the subluminal observers. This creates a physical difference between subluminal and superluminal observers.

Since the Galilean principle of relativity stating that all inertial frames are equivalent does not hold in the $1+3$ dimensional spacetime, we propose a quantum version of the principle of relativity. We postulate that the existence or non-existence of a local and deterministic mode of description of any process should not depend on the choice of the inertial reference frame. For example, if there is no local deterministic mechanism (or 'element of reality') behind the particle decay in figure 1(b) in the past world-line of B in one frame, there should be no such mechanism in any other frame. This way all the conclusions of the previous sections are still valid, while we allow for the two families of observers to be physically distinguishable.

Lastly, we would like to propose a different interpretation of the relation between spacetime intervals in subluminal and superluminal reference frames, given by (16). Let us notice, that the common signs of individual terms on the right-hand side of the equation (16) suggest that the temporal coordinate $\mathrm{d} t^{\prime}$ should have the same properties as $\mathrm{d} y^{\prime}$ and $\mathrm{d} z^{\prime}$. The quantity $\mathrm{d} t^{\prime}$ can be identified as a temporal coordinate, because its axis $t^{\prime}$ must coincide with the world line of the superluminal observer. This suggests, that the remaining coordinates, $y^{\prime}$ and $z^{\prime}$ are also temporal, and there is only a single spatial dimension in a superluminal frame of reference, $x^{\prime}$. Within such an interpretation, the interval in the $n+m$ dimensional spacetime, defined as: $\mathrm{d} s^{2} \equiv c^{2} \sum_{i=1}^{n} \mathrm{~d} t_{i}^{2}-\sum_{i=1}^{m} \mathrm{~d} r_{i}^{2}$ changes its sign for the superluminal coordinate transformation, and the two perpendicular spatial coordinates change their character transforming the $n+m$ dimensional spacetime into the $m+n$ dimensional one.

Such a disturbing property of superluminal observers, not only explains a physical difference between them and subluminal reference frames, but also offers an interesting insight into the origin of the wave properties of matter with a novel interpretation of the Huygens principle. According to that principle, any point, at which a particle wave arrives becomes the origin of a new spherical 'mater wave'. Since all known matter (and light) follows this principle, it appears as if all physical objects were compelled to propagate in all directions of space from any point they visit. But this peculiar behavior observed from a superluminal reference frame appears to be as if all object were forced to move symmetrically in all 'directions of time', which, in a some way, sounds more appropriate.

The $1+3$ dimensional Lorentz transformation between two subluminal observers is obtained from (8) by replacing $V x$ with $\boldsymbol{V} \cdot \boldsymbol{r}$, where $\boldsymbol{V}$ is an arbitrary subluminal velocity and $\boldsymbol{r}=(x, y, z)$. It can be written in the coordinate-independent form as: 


$$
\begin{aligned}
\boldsymbol{r}^{\prime} & =\boldsymbol{r}-\frac{\boldsymbol{V} \cdot \boldsymbol{r}}{V^{2}} \boldsymbol{V}+\frac{\frac{V \cdot \boldsymbol{r}}{V^{2}}-t}{\sqrt{1-V^{2} / c^{2}}} \boldsymbol{V}, \\
c t^{\prime} & =\frac{c t-\frac{V \cdot \boldsymbol{r}}{c}}{\sqrt{1-V^{2} / c^{2}}} .
\end{aligned}
$$

The inverse transformation is obtained by substituting $\boldsymbol{V} \rightarrow-\boldsymbol{V}$, as well as $\boldsymbol{r} \leftrightarrow \boldsymbol{r}^{\prime}$ and $t \leftrightarrow t^{\prime}$.

Similar generalization can be carried out for the superluminal transformations (9). By replacing $V x$ in (9) with $\boldsymbol{V} \cdot \boldsymbol{r}$, we obtain the coordinate-independent transformation between a subluminal reference frame $(t, \boldsymbol{r})$ and a superluminal one $\left(\boldsymbol{t}^{\prime}, x^{\prime}\right)$ moving with a superluminal velocity $\boldsymbol{V}$ :

$$
\begin{aligned}
x^{\prime} & =\frac{V t-\frac{V \cdot \boldsymbol{r}}{V}}{\sqrt{V^{2} / c^{2}-1}}, \\
c \boldsymbol{t}^{\prime} & =\boldsymbol{r}-\frac{\boldsymbol{V} \cdot \boldsymbol{r}}{V^{2}} \boldsymbol{V}+\frac{\frac{V \cdot \boldsymbol{r}}{V c}-\frac{c t}{V}}{\sqrt{V^{2} / c^{2}-1}} \boldsymbol{V} .
\end{aligned}
$$

The inverse transformation is obtained by reversing the above set of linear equations. It is equivalent to substituting $\boldsymbol{V} \rightarrow-\boldsymbol{V}$, as well as $\boldsymbol{r} \leftrightarrow \boldsymbol{t}^{\prime}$ and $t \leftrightarrow x^{\prime}$. For the infinite speed limit $V \rightarrow \infty$, the above formulas reduce to:

$$
\begin{aligned}
x^{\prime} & =c t, \\
c \boldsymbol{t}^{\prime} & =\boldsymbol{r},
\end{aligned}
$$

regardless of the direction of the velocity $V$.

\section{Summary}

We argue that ruling out a superluminal family of observers from special relativity, regardless whether such observers exist or not, is not necessary; it leads to a classical description of a particle moving along a well-defined single trajectory. In contrast, if one keeps both subluminal and superluminal solutions then non-deterministic behavior and non-classical motion of particles arise as a natural consequence.

The superluminal solutions appear quite naturally in general relativity. For instance a Schwarzschild solution to Einstein equations written in Schwarzschild coordinates has a peculiar property that time and radial coordinates change their metric signs at the event horizon. This is normally dismissed by arbitrarily stating that the Schwarzschild solutions only make sense above the event horizon, although written in (freely falling) Kruskal coordinates, they are smooth at the horizon. In order to resolve this puzzle we point out that Schwarzschild coordinates correspond to stationary observers placed at fixed distances from the horizon. Such observers can be subluminal only above the horizon, and under the horizon they would require superluminal motions. The sign flip in the metric therefore signifies the transition from a subluminal to a superluminal family of stationary observers residing under the event horizon in a fixed distance from the singularity.

We believe that our approach is more than a mathematical exercise and, if taken seriously, it may offer new valuable insights into deep connections between quantum theory and special relativity.

\section{References}

[1] Derivation of Lorentz transformation without the assumption of the constancy of the speed of light was independently discovered in Ignatowsky W 1910 Arch. Math. Phys. 17 as well as

Frank P and Rothe H 1911 Ann. der Phys. 34825 and later in

Szymacha A 1997 Przestrzeń i Ruch (Warsaw: University of Warsaw) Here we present a modified and extended version of this derivation

[2] Machildon L, Antippa A F and Everett A E 1983 Can. J. Phys. 61256

[3] Machildon L, Antippa A F and Everett A E 1983 Phys. Rev. D 271740

[4] Sutherland R I and Shepanski J R 1986 Phys. Rev.D 332896

[5] Dragan A 2008 arXiv:quant-ph:0806.4875 